This document is confidential and is proprietary to the American Chemical Society and its authors. Do not copy or disclose without written permission. If you have received this item in error, notify the sender and delete all copies.

\title{
Dynamic Behavior of Single Fe Atoms Embedded in Graphene
}

\begin{tabular}{|r|l|}
\hline Journal: & The Journal of Physical Chemistry \\
\hline Manuscript ID & Draft \\
\hline Danuscript Type: & Article \\
\hline Complete List of Authors: & $\begin{array}{l}\text { Markevich, Alexander; University of Nottingham } \\
\text { Baldoni, Matteo; University of Nottingham } \\
\text { Warner, Jamie; University of Oxford, Materials } \\
\text { Kirkland, Angus; University of Oxford, Department of Materials } \\
\text { Besley, Elena; University of Nottingham, Chemistry }\end{array}$ \\
\hline
\end{tabular}

\section{SCHOLARONE ${ }^{m}$}

Manuscripts 


\title{
Dynamic Behavior of Single Fe Atoms
}

\section{Embedded in Graphene}

\author{
Alexander V. Markevich, ${ }^{\dagger}$ Matteo Baldoni, ${ }^{\dagger}$ Jamie H. Warner ${ }^{+\dagger},{ }^{\dagger}$ Angus I. Kirkland, ${ }^{,}$ \\ and Elena Besley ${ }^{+* *}$ \\ ${ }^{\dagger}$ School of Chemistry, University of Nottingham, University Park, Nottingham NG7 2RD, UK, \\ ${ }^{t}$ Department of Materials, University of Oxford, Parks Road, Oxford OX1 3PH, UK \\ *Corresponding author: elena.besley@nottingham.ac.uk
}




\begin{abstract}
The effect of electron irradiation on the dynamic behavior of $\mathrm{Fe}$ atoms, embedded into monovacancy (Fe@MV) and di-vacancy (Fe@DV) defects in graphene, has been investigated using $a b$ initio molecular dynamics. This study reveals the detailed mechanisms of transformation and migration of the Fe@MV and Fe@DV defects in graphene recently observed in aberrationcorrected high-resolution transmission electron microscopy (AC-HRTEM) experiments [Nano Lett. 2013, 13, 1468]. An important atomic-scale insight into the dynamics of atomic Fe on graphene, unavailable to AC-HRTEM observations, has been provided. It was found that structural changes of the studied defects are induced by electron impacts on carbon atoms bonded to Fe. The threshold energies for ejection of these carbon atoms are significantly lower compared to that in pristine graphene. For electron impacts with the sub-threshold transferred energies, migration of the defects and flipping of $\mathrm{Fe}$ atoms between different sides of the graphene plane can occur. The stability of the Fe@MV defect under electron irradiation strongly depends on the substrate side position of Fe atom with respect to the direction of the electron beam. The Fe@DV $\rightarrow$ Fe@MV transformations take place spontaneously in the presence of carbon ad-atoms, which are available in abundance on graphene in AC-HRTEM. The present study facilitates a greater general understanding of the dynamic behavior of substitutional metal atoms in graphene.
\end{abstract}




\section{INTRODUCTION}

Interactions between graphene and metals have been extensively studied to aid the development of new graphene functionalities for technological applications, understanding the properties of graphene-metal electrical contacts, ${ }^{1,2}$ catalytic growth of graphene on metallic surfaces, ${ }^{3-5}$ and production of fuel cells, ${ }^{6-8}$ to name a few. Adsorption of metal atoms on graphene is considered to be a promising approach to manipulation of its electronic and magnetic properties. Doping graphene with free charge carriers has been predicted theoretically and confirmed experimentally for various metallic adsorbates. ${ }^{9-14}$ However, metal ad-atoms on graphene typically have low activation barriers for diffusion and therefore are very mobile at room temperatures. ${ }^{9,15,16}$ A lot of attention has been recently devoted to substitutional metal impurities in graphene. Such structures have been predicted to be very stable and can induce magnetism, which makes them of significant importance for applications in spintronics. ${ }^{16}$ Metal atoms embedded into the graphene lattice have been also predicted to be efficient catalysts for oxygen reduction ${ }^{17}$ and $\mathrm{CO}$ oxidation reactions. ${ }^{18}$

Incorporation of metal atoms into substitutional sites in graphene and carbon nanotubes has been realized experimentally and can potentially be performed with nearly atomic precision. ${ }^{19-22}$ In these experiments, high energy particles (electrons, ions) have been used to create vacancy defects in graphene which act as trapping centers for metal atoms. An interesting dynamic behavior of Fe atoms embedded in graphene mono- and di-vacancies (labelled as Fe@MV and Fe@DV) has been shown in real time using aberration-corrected high-resolution transmission electron microscopy (AC-HRTEM) at $80 \mathrm{kV}$ accelerating voltage. ${ }^{22}$ The authors observed a reversible Fe@MV $\leftrightarrow \mathrm{Fe} @ \mathrm{DV}$ defect transformation as well as migration of these defects with no site symmetry change. Theoretical studies of $\mathrm{Fe}$ atoms embedded into mono- and di-vacancies 
in graphene predict a barrier to migration of $3.6 \mathrm{eV}$ for Fe@MV and about 5 eV for Fe@DV thus suggesting a high stability of these defects. ${ }^{16}$ Hence, the experimentally observed defect dynamics has been proposed to be caused by electron beam irradiation. A comprehensive understanding of the dynamics of substitutional metal atoms in graphene is essential for achieving control over the fabrication process and creation of graphene-metal nanostructures with desired properties. In this work, ab initio molecular dynamics (AIMD) studies have been performed to investigate the effects of electron irradiation on the dynamics of the Fe@MV and Fe@DV complexes in graphene.

\section{COMPUTATIONAL METHODS}

Density functional theory (DFT) calculations have been performed using the PBE form of the exchange-correlation functional ${ }^{23}$ and Troullier-Martins pseudopotentials ${ }^{24}$ as implemented in the SIESTA code. ${ }^{25}$ For the valence states double- $\zeta$ basis sets of localized orbitals including polarization functions were used. The charge density was represented on a real-space grid with an energy cut-off of $250 \mathrm{Ry}$. Graphene layers were modelled using orthorhombic supercells consisting of 160 carbon atoms. A vacuum layer of $20 \AA$ was included in the direction normal to the graphene plane to avoid interactions between the graphene sheet and its replicas. The structures were optimized using a conjugate-gradient algorithm with the convergence criterion for the force of $0.02 \mathrm{eV} / \AA$. In all calculations spin polarization of the valence states has been taken into account. For analysis of structural and electronic properties of the defects the Brillouin zone was sampled using a $3 \times 3 \times 1 k$-points grid generated within the Monkhorst-Pack scheme. ${ }^{26}$ AIMD simulations were performed at the $\Gamma$ point with a time step of 0.5 fs. To account for the dispersion interactions the Grimme's semiempirical potential was used. ${ }^{27}$ The 
threshold energies for irradiation-induced transformations were calculated by varying the amount of the transferred energy to the target atom with $0.1 \mathrm{eV}$ intervals.

\section{RESULTS AND DISCUSSION}

\subsection{Structural and electronic properties of Fe@MV and Fe@DV}

Atomic structures of the Fe@MV and Fe@DV complexes are shown in Figure 1, where a single Fe atom substitutes missing carbon atom(s) and passivates the undercoordinated atoms of the native mono- and di-vacancy defects. The calculated structural parameters and binding energies are presented in Table 1 and are in good agreement with previously reported results. ${ }^{16}$ The binding energies have been calculated with respect to a reconstructed vacancy defect with a distant $\mathrm{Fe}$ atom. As the $\mathrm{Fe}$ atom has a larger atomic radius as compared to a carbon atom, once embedded into the mono-vacancy it is significantly displaced out of the graphene plane, by about $1.45 \AA$ A. It also induces a considerable out-of-plane shift of the neighbouring carbon atoms (Figure 1c). In the case of Fe@DV, the out-of-plane distortion of the surrounding graphitic lattice is much smaller (Figure 1d), and the elevation of the Fe atom above the graphene surface is only $0.57 \AA$. 


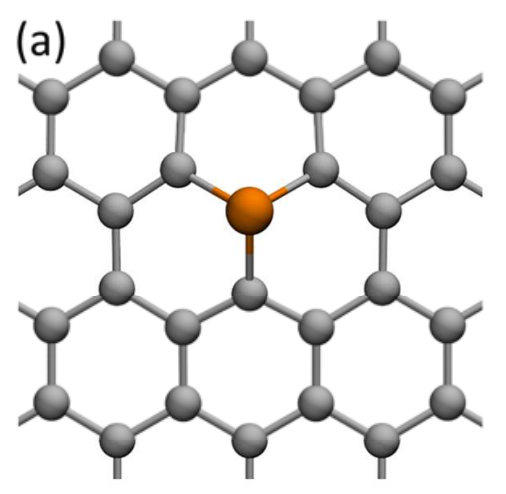

(c)

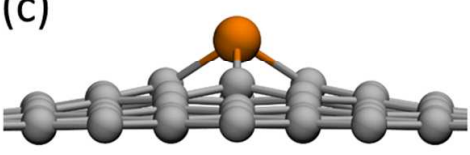

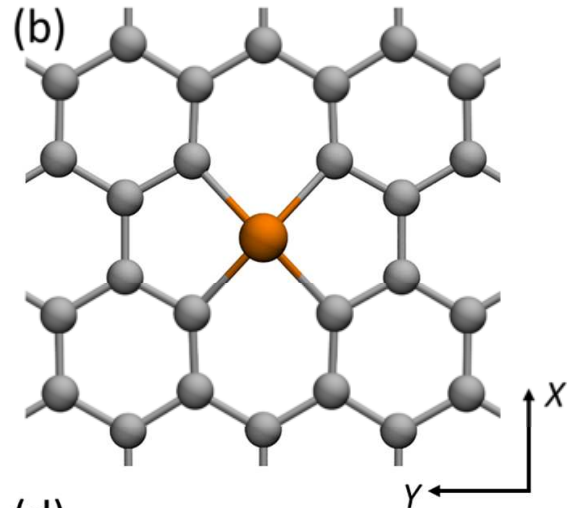

(d)

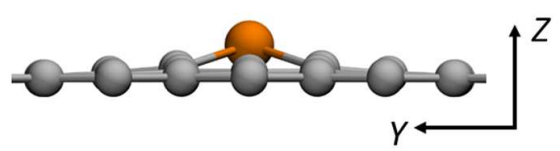

Figure 1. Optimized atomic structures of Fe@MV (left) and Fe@DV defects (right): (a) and (b) top view, (c) and (d) side view. Carbon atoms are represented by grey spheres, Fe atoms are shown as larger orange spheres.

Table 1. PBE DFT values of the Fe-C bond length $d_{\mathrm{Fe}-\mathrm{C}}$, elevation of the $\mathrm{Fe}$ atom above the graphene surface $h$, binding energy $E_{\mathrm{b}}$, and magnetic moment $M$ for Fe@MV and Fe@DV structures.

\begin{tabular}{lllll} 
& $d_{\mathrm{Fe}-\mathrm{C}}, \AA$ & $h, \AA$ & $E_{\mathrm{b}}, \mathrm{eV}$ & $M, \mu_{\mathrm{B}}$ \\
\hline Fe@MV & 1.77 & 1.45 & 7.58 & 0 \\
Fe@DV & 1.97 & 0.57 & 6.35 & 3.6
\end{tabular}

The Fe@MV and Fe@DV defects have very different electronic properties. While Fe@MV is not magnetic, the Fe@DV has a magnetic moment of $3.6 \mu_{\mathrm{B}}$. Density of states (DOS) calculations of Fe@MV (Figure 2a) show the absence of electronic states at the Fermi level, $E_{\mathrm{F}}$. The DOS also goes to zero at about $-0.5 \mathrm{eV}$ and $0.2 \mathrm{eV}$ with respect to $E_{\mathrm{F}}$. Inside this energy 
interval there are, however, two sharp peaks that indicate the presence of occupied and unoccupied electronic bands with low dispersion. The electronic structure of Fe@DV is more complex. The DOS shows a number of spin-polarized states close to the Fermi level and nonzero DOS at $E_{\mathrm{F}}$ (Figure 2b). Such states have been demonstrated to have a metal-carbon bonding character. $^{16}$
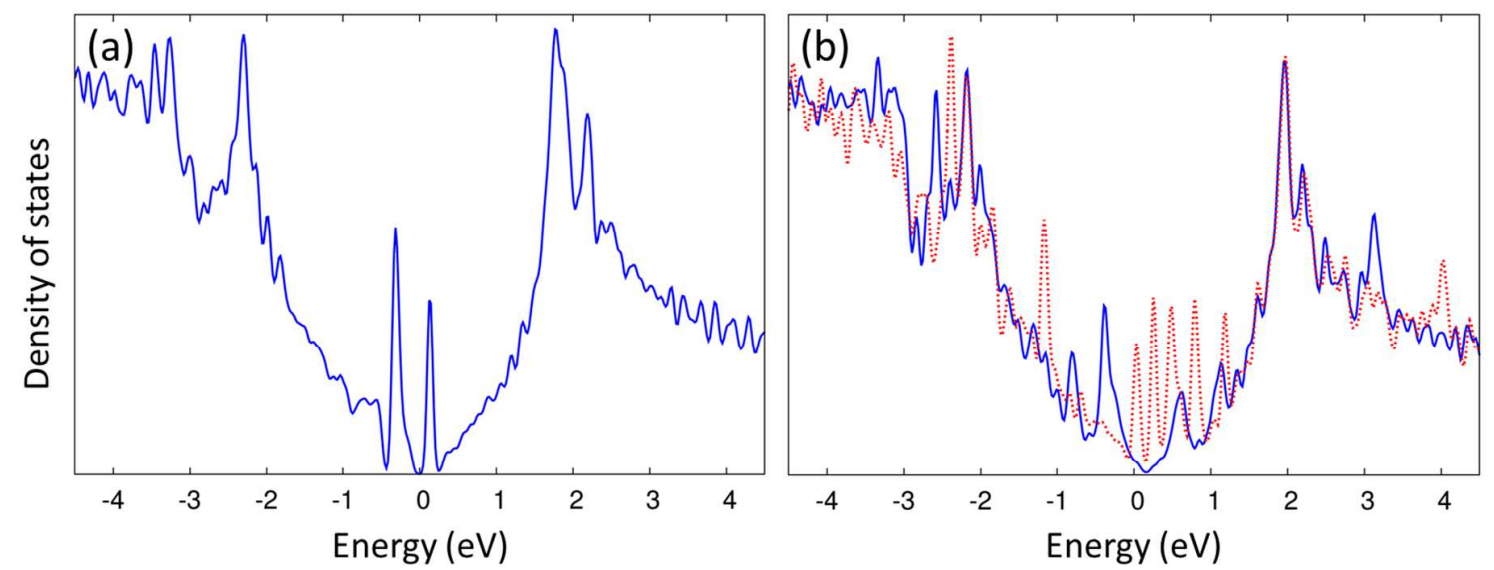

Figure 2. Calculated DOS of Fe@MV (a) and Fe@DV (b). Solid blue lines and dotted red lines correspond to the majority and minority carrier spin respectively. Energy scale zero corresponds to the position of the Fermi level.

\subsection{Dynamics of Fe@MV and Fe@DV under electron ir radiation}

To investigate the effects of electron irradiation on structural changes of Fe@MV and Fe@DV a well-established methodology ${ }^{28-31}$ has been followed, where molecular dynamics simulations are used to model the evolution of a system with time after an elastic collision between a highenergy electron and a nucleus took place. In such collision, direction and magnitude of the momentum transferred to a nucleus of a sample depend on the electron scattering angle $\theta$. In spherical coordinate system, direction of the transferred momentum can be defined by the polar 
angle $\Omega=\left(180^{\circ}-\theta\right) / 2$, counted from the direction of the incident electron, and azimuthal angle $\varphi$. Figure 3 shows orientation of graphene layer and direction of the electron beam adopted in the present simulations. The maximum kinetic energy that can be transferred to a nucleus by an elastically scattered electron corresponds to $\theta=180^{\circ}$. In this case $\Omega=0^{\circ}$ and the transferred momentum is directed perpendicular to the graphene plane. For $80 \mathrm{keV}$ electrons the maximum kinetic energy transferred to $\mathrm{Fe}$ and $\mathrm{C}$ atoms is $T_{\max }(\mathrm{Fe})=3.4 \mathrm{eV}$ and $T_{\max }(\mathrm{C})=15.8 \mathrm{eV}$, respectively. Our calculations show that scattering of $80 \mathrm{keV}$ electrons by $\mathrm{Fe}$ atoms is unlikely to induce significant structural changes to the Fe@MV or Fe@DV defects. However, the $\mathrm{C}$ atoms covalently bonded to Fe receive sufficient energy to break both $\mathrm{Fe}-\mathrm{C}$ and $\mathrm{C}-\mathrm{C}$ covalent bonds.

(a)

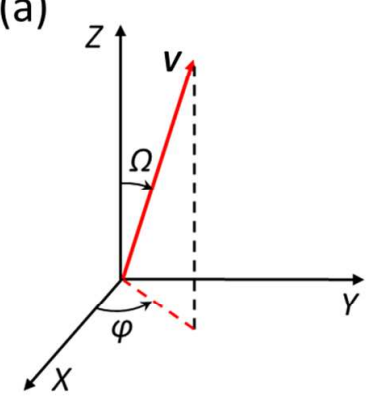

(b)

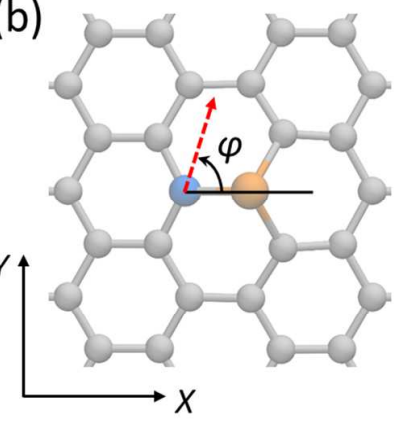

(c)

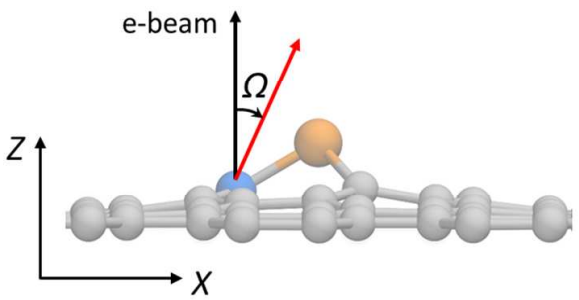

Figure 3. The direction of the momentum transferred to the $\mathrm{C}$ atom as a result of a collision with a high energy electron is shown with the red arrow and is defined by the polar angle $\Omega$, measured from the direction of the incident electron (along $Z$ axis), and the azimuthal angle $\varphi$. (b) and (c) show orientation of the axes and emission angles of the carbon atom for the Fe@MV defect in graphene.

To evaluate the stability of the Fe@MV defect under electron irradiation, the threshold energy required for emission of the target carbon atom (shown in blue in Figures 3b, 3c) has been 
calculated for the case of $\theta=180^{\circ}$, i.e. when the transferred momentum is directed perpendicular to graphene plane. Since the out-of-plane character of the defect cannot be distinguished in ACHRTEM images two possible configurations have been considered, when Fe atoms are displaced either above, as shown in Figure 3c, or below graphene plane. For the case shown in Figure 3c, where the $\mathrm{Fe}$ atom is located above graphene plane, the threshold energy for ejection of the target carbon atom is $T_{\mathrm{e}}=15 \mathrm{eV}$. If the defect is formed below graphene plane, the threshold energy increases to $T_{\mathrm{e}}=17 \mathrm{eV}$.

Knowing the values of the threshold energy the cross section, $\sigma_{\mathrm{e}}$, for the transformation of Fe@MV to Fe@DV under irradiation with $80 \mathrm{keV}$ electrons can be estimated using the McKinley-Feshbach formula. ${ }^{32}$ Taking into account the out-of-plane lattice vibrations with a Debye temperature of $1287 \mathrm{~K}^{30}$ and the number of equivalent $\mathrm{C}$ atoms bonded to $\mathrm{Fe}$, we have obtained the cross section for the Fe@MV $\rightarrow$ Fe@DV transformation of $\sigma_{\mathrm{e}}=21.2$ barn $(\mathrm{Fe}$ above the graphene plane) and $\sigma_{\mathrm{e}}=5.5$ barn (Fe below the graphene plane). The cross section was assumed to be isotropic with respect to the direction of emission, a widely used approximation in evaluation of stability of the structures under electron irradiation. ${ }^{33,34}$ These results suggest that the probability for the Fe@MV $\rightarrow \mathrm{Fe} @ \mathrm{DV}$ transformation to occur under electron irradiation at $80 \mathrm{keV}$ is sufficiently large for the transformation to be observed in ACHRTEM. For example, for the experimentally used beam current density of $10^{6} \mathrm{e} / \mathrm{nm}^{2} / \mathrm{s}$ the cross section of 21.2 barn corresponds to about one transformation in 8 minutes.

It has been further found that electron irradiation can induce migration of Fe@MV to the adjacent lattice site when the Fe atom is displaced along the direction of the incident electron beam. The migration occurs when the energy transferred to carbon atom is in the range of 13.5$14.9 \mathrm{eV}$. Figure 4a shows snapshots of AIMD simulation for the impact energy of $14 \mathrm{eV}$. The 
ejection of carbon atom from the defect results in the formation of an intermediate Fe@DV structure. Due to the strong interaction with the metal atom the ejected carbon atom gains an inplane component to its velocity and moves towards the opposite site of the defect where it is reincorporated back into the lattice. This effectively results in a swap of $\mathrm{Fe}$ and $\mathrm{C}$ atom positions in the lattice and a shift of the defect by one lattice site. A similar migration mechanism has been recently demonstrated for the $\mathrm{Si} @ \mathrm{MV}$ defect in graphene. ${ }^{34}$ A pathway for the migrating $\mathrm{C}$ atom depends strongly on the amount of the transferred energy. For the impact energy of $14 \mathrm{eV}$, the $\mathrm{C}$ atom moves over the Fe following a high trajectory, which becomes lower if the value of the transferred energy is decreased. Eventually, the ejected $\mathrm{C}$ atom forms a bond with $\mathrm{Fe}$ again leading to an intermediate structure comprising of $\mathrm{Fe} @ \mathrm{DV}$ with the $\mathrm{C}$ ad-atom, as shown in Figure 4a. In all our simulations such structures spontaneously recombine to Fe@MV suggesting that $\mathrm{Fe} @ \mathrm{DV}+\mathrm{C}$ ad-atom is a metastable configuration. It has been also observed that during migration of the defect the Fe atom typically flips to the opposite site of the graphene plane (Figure 4).
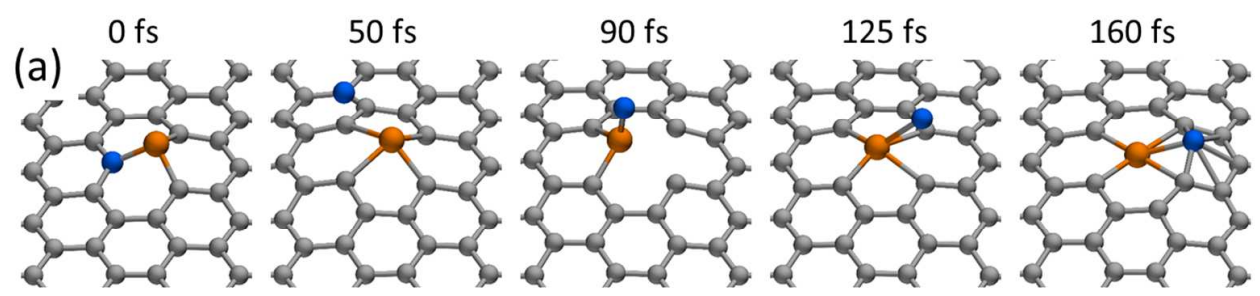

$225 \mathrm{fs}$

(b)
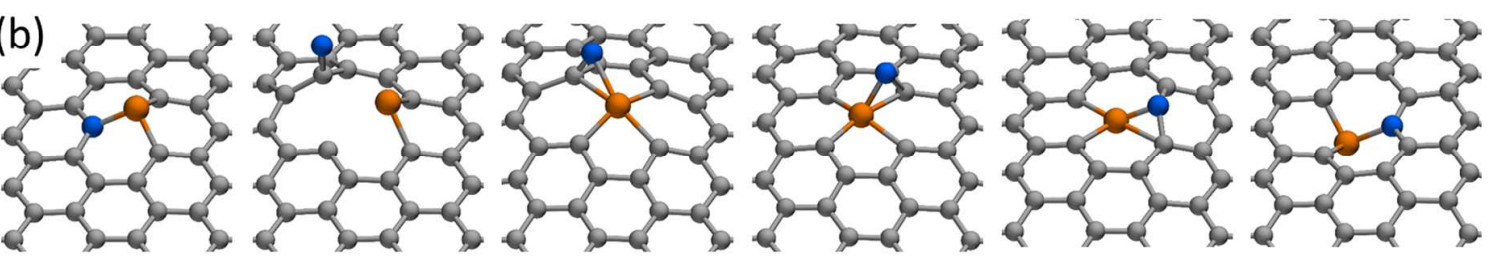

Figure 4. Snapshots of AIMD simulations showing migration of the Fe@MV defect. In (a) the carbon atom shown in blue has been given a momentum that corresponds to a transferred energy 
of $14 \mathrm{eV}$ and directed perpendicular to the graphene plane. In (b) the direction and the magnitude of the transferred momentum corresponds to a collision event with an $80 \mathrm{keV}$ electron scattered at an angle, $\theta=150^{\circ}$. The emission angles of the carbon atom are $\Omega=15^{\circ}$ and $\varphi=60^{\circ}$ (see Figure 3). The timing is shown in femtoseconds (fs) after the collision event and is applicable to both (a) and (b).

In the simulations described above it has been assumed that the direction of the momentum transferred to a $\mathrm{C}$ atom is normal to graphene plane for different values of the transferred energy. In reality, however, direction and magnitude of the transferred momentum are functions of the electron scattering angle $\theta$, and the impacted atom always has an in-plane component of its velocity, except at $\theta=180^{\circ}$ (see Figure 3). Therefore, the impacts of $80 \mathrm{keV}$ electrons, scattered with angles of $150^{\circ}$ and $160^{\circ}$, on the $\mathrm{C}$ atoms bonded to Fe have been also considered. The energy transferred to carbon atom in such collisions is $T=14.7 \mathrm{eV}$ for $\theta=150^{\circ}$ and $T=15.3 \mathrm{eV}$ for $\theta=160^{\circ}$. It has been found that all impacts corresponding to $\theta=150^{\circ}$ and $\varphi=60^{\circ}, 90^{\circ}, 150^{\circ}$ and $180^{\circ}$ result in the migration of the $\mathrm{Fe} @ \mathrm{MV}$ defect. Figure $4 \mathrm{~b}$ shows the migration of the defect for the case of $\varphi=60^{\circ}$. For $\varphi=180^{\circ}$ the in-plane component of the initial velocity of the $\mathrm{C}$ atom is directed outwards from the defect. The interaction with the $\mathrm{Fe}$ atom changes the direction of the carbon atom motion and the resulting migration path is very similar to the one shown in Figure 4a. In simulations with $\varphi=0^{\circ}, 30^{\circ}$ and $120^{\circ}$ the ejected $\mathrm{C}$ atom returns to the same lattice position, but the $\mathrm{Fe}$ atom flips to the opposite site of the graphene plane. Interestingly, for an electron scattering angle of $160^{\circ}$ the energy transferred to the $\mathrm{C}$ atom is higher than the calculated emission threshold energy of $T_{\mathrm{e}}=15 \mathrm{eV}$. However, migration of $\mathrm{Fe} @ \mathrm{MV}$ for $\varphi=60^{\circ}, 90^{\circ}$ and $180^{\circ}$ and recombination of the ejected carbon to the same lattice 
site for $\varphi=30^{\circ}$ are still observed. These results indicate a strong anisotropy of the cross sections for emission and migration of $\mathrm{Fe} @ \mathrm{MV}$ with respect to the emission angles. When Fe atoms are displaced against the direction of the incident electrons no migration of $\mathrm{Fe} @ \mathrm{MV}$ is observed.

Our calculations show that the stability of the Fe@MV defect under electron irradiation, for both defect migration and carbon atom emission, strongly depends on the direction of the out-ofplane displacement of the $\mathrm{Fe}$ atom with respect to the direction of the incident electrons. The energy barrier for the transition of the Fe atom through the graphene sheet was calculated to be $2.3 \mathrm{eV}$, assuming that the saddle point corresponds to the configuration where the Fe atom positioned at the center of the graphene mono-vacancy. According to our AIMD simulations, for this process to be induced by electron irradiation at $80 \mathrm{keV}$ the energy transferred to the Fe atom should be higher than $7 \mathrm{eV}$. For comparison, the maximum energy that can be transferred to $\mathrm{Fe}$ due to a collision with a $150 \mathrm{keV}$ electron is about $6.8 \mathrm{eV}$. Such electrons, however, will quickly cause damage to the graphene lattice by alternative mechanisms. These results suggest that transition between different out-of-plane orientations of Fe@MV is unlikely to occur due to thermal effects or direct collisions between the Fe atom and the high-energy electrons. During migration of Fe@MV the Fe atom typically flips from above the plane to below the plane site (Figure 4), this can occur without any in-plane migration and for the impact energies down to $13.3 \mathrm{eV}$. However, the opposite movement of Fe in Fe@MV structure has not been observed in our simulations suggesting that such transitions, if possible, have rather low probability. This suggest that under $80 \mathrm{keV}$ electron irradiation, the $\mathrm{Fe} @ \mathrm{MV}$ defects preferably adopt a configuration with the Fe atom displaced against the direction of the beam, which is more stable to the irradiation induced transformations. 
For Fe@DV our calculations yield threshold energies for emission of the $\mathrm{C}$ atom of $T_{\mathrm{e}}=15.7$ $\mathrm{eV}$ if the Fe atom is displaced along the direction of the incident electrons, and $T_{\mathrm{e}}=16.3 \mathrm{eV}$ for Fe displacement against the direction of the electron beam. Taking into account that there are four equivalent $\mathrm{C}$ atoms bonded to $\mathrm{Fe}$ the cross sections for the $\mathrm{Fe} @ \mathrm{DV} \rightarrow \mathrm{Fe} @ \mathrm{TV}$ transformation was estimated to be $\sigma_{\mathrm{e}}=18.5$ barn for $T_{\mathrm{e}}=15.7 \mathrm{eV}$ and $\sigma_{\mathrm{e}}=12.4$ barn for $T_{\mathrm{e}}=$ 16.3 $\mathrm{eV}$. The difference in the values of the threshold energies for the up and down configurations is significantly smaller than in the case of Fe@MV, which is expected due to the smaller out-of-plane distortion of the Fe@DV defect. Moreover, it has been found that the energy barrier for the Fe atom flipping through the graphene plane is only $0.2 \mathrm{eV}$. Such a low energy barrier suggests that Fe@DV can easily switch between up and down configurations at room temperature.

While our calculations show that Fe@DV $\rightarrow$ Fe@TV transformations can be in principle promoted by an $80 \mathrm{keV}$ electron beam, such transformations have not been previously observed experimentally. The out-of-plane lattice vibrations can be locally suppressed by the presence of the heavy Fe atom, which will considerably reduce their contribution to the kinetic energy transfer at the impacted atom. For comparison, within the static lattice approximation the transformation of Fe@DV to Fe@TV will have a cross section of 1.2 barn and 0 barn for the emission threshold energies of $15.7 \mathrm{eV}$ and $16.3 \mathrm{eV}$, respectively. There is also a possibility that recombination of $\mathrm{Fe} @ \mathrm{DV}$ with $\mathrm{C}$ ad-atoms, which are always present in experiment, occurs more often than emission of $\mathrm{C}$ atom. The experimental statistics for the Fe@DV defects is, however, rather limited, ${ }^{22}$ which makes any direct comparison with theoretical results difficult.

For impacts with energies below $15.7 \mathrm{eV}$ no resultant transformations of Fe@DV have been observed, except flipping of the Fe atom to the opposite site of the graphene plane. However, 
simulations of impacts that correspond to different electron scattering angles indicate the possibility of the induced migration of the Fe@DV defect. Figure 5 shows snapshots of AIMD simulation for the impact corresponding to $\theta=150^{\circ}$ and $\varphi=-30^{\circ}$. The migration mechanism is similar to that of $\mathrm{Fe} @ \mathrm{MV}$ and occurs via the ejection of a $\mathrm{C}$ atom and formation of the intermediate metastable structure comprising of Fe@TV with a C ad-atom. Migration of the defect has been also observed in simulations for $\theta=150^{\circ}, \varphi=-60^{\circ}$ and $\theta=160^{\circ}, \varphi=-15^{\circ}$. In many calculations the intermediate structure shown in Figure 5 at 110 fs has been observed. However, after some time the ejected $\mathrm{C}$ atoms returns to the initial lattice position.
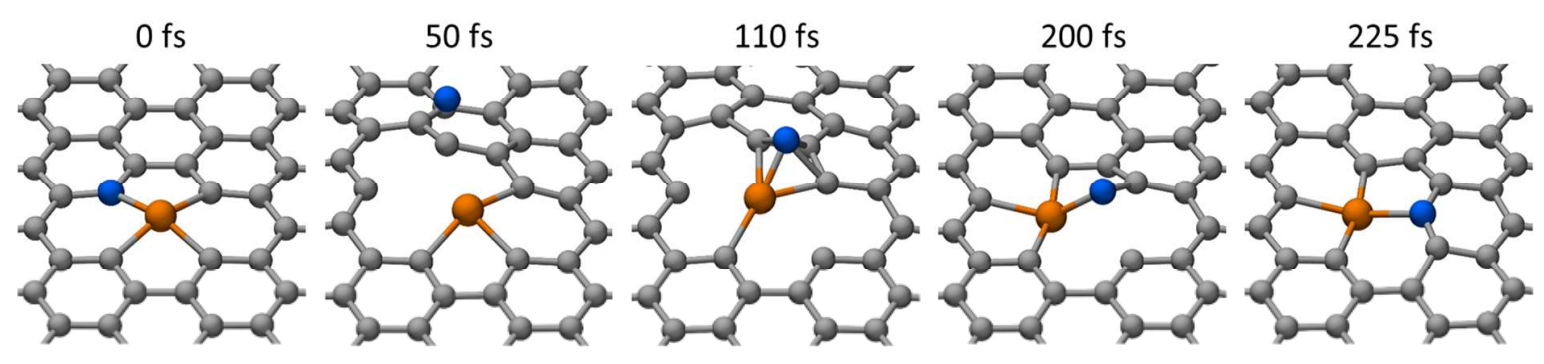

Figure 5. Snapshots of AIMD simulations showing migration of the Fe@DV. The carbon atom shown in blue has been given a momentum corresponding to a collision event with an $80 \mathrm{keV}$ electron scattered at an angle $\theta=150^{\circ}$. The emission angles of the carbon atom are $\Omega=15^{\circ}$ and $\varphi=-30^{\circ}$ (see Figure 3).

Along with the migration of the defect and emission of the $\mathrm{C}$ atom other structural transformations have been observed. Figure 6a shows the reconstructed Fe@DV formed after impact with $\theta=160^{\circ}$ and $\varphi=-30^{\circ}$. Similar reconstructions of Fe $@ \mathrm{DV}$ have been experimentally observed in the TEM at $80 \mathrm{keV} .^{22}$ In Figure $6 \mathrm{~b}$ another interesting outcome of molecular dynamics simulation is shown where the ejected $\mathrm{C}$ atom lands on graphene away from the defect. 
In this case, thermodynamically driven recombination of Fe@TV with the ejected atom can result in formation of some of the other reconstructions of Fe@DV that have been observed experimentally.
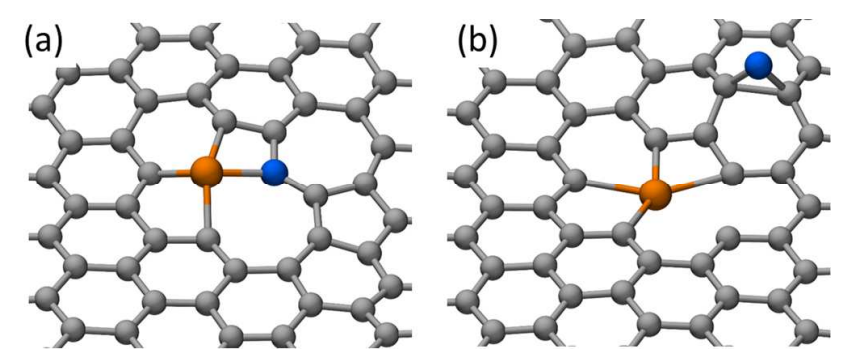

Figure 6. Atomic configurations observed in molecular dynamics simulations of structural transformations of $\mathrm{Fe} @ \mathrm{DV}$ as a result of a momentum transfer from an $80 \mathrm{keV}$ electron. The emission angles of the carbon atom are $\Omega=10^{\circ}$ and $\varphi=-30^{\circ}$ (a) and $\Omega=5^{\circ}$ and $\varphi=0^{\circ}$ (b).

\section{CONSCLUSIONS}

In the present work the effect of electron irradiation on structural changes of Fe@MV and Fe@DV defects in graphene has been investigated using ab initio molecular dynamics. It is shown that impacts of high-energy electrons on the carbon atoms adjacent to Fe promotes various structural transformations including ejection of the $\mathrm{C}$ atoms, migration of the defects and transition of $\mathrm{Fe}$ atoms between different sides of the graphene plane. The calculated threshold energies of irradiation-induced transformations suggest that they will be feasible for electron energies of 80-100 keV, which are typically used in AC-HRTEM experiments. Under such irradiation conditions the effect of electron impacts on the Fe atoms is insignificant. It has also been found that Fe@DV is not stable against recombination with $\mathrm{C}$ ad-atoms. 
Stability of substitutional metal atoms in graphene under irradiation depends on many factors, such as the type of the atom and structure of the defect, accelerating voltage and current density of the electron beam. Theoretical approach used in the present work can be employed to predict the behavior of different metal-graphene complexes under electron irradiation. Moreover, it can reveal the out-of-plane structural transformations that cannot be observed in AC-HRTEM experiments. This modelling work facilitates greater understanding of the dynamics of metal atoms embedded into the graphene lattice and, in particular, the effect of electron irradiation on transformation and migration mechanisms of these defects.

\section{ACKNOWLEDGMENTS}

AVM, MB and EB acknowledge the High Performance Computing (HPC) Facility at the University of Nottingham for providing computational time. EB acknowledges an ERC Consolidator Grant for financial support. 


\section{Refer ences}

(1) Gong, C.; McDonnell, S.; Qin, X.; Azcatl, A.; Dong, H.; Chabal, Y. J.; Cho, K.; Wallace, R. M. Realistic Metal-Graphene Contact Structures. ACS Nano 2014, 8, 642-649.

(2) Leong, W. S.; Nai, C. T.; Thong, J. T. L. What Does Annealing Do to Metal-Graphene Contacts? Nano Lett. 2014, 14, 3840-3847.

(3) Reina, A.; Jia, X.; Ho, J.; Nezich, D.; Son, H.; Bulovic, V.; Dresselhaus, M. S.; Jing, K. Large Area, Few-Layer Graphene Films on Arbitrary Substrates by Chemical Vapor Deposition. Nano Lett. 2009, 9, 30-35.

(4) Voloshina, E.; Dedkov, Y. Graphene on Metallic Surfaces: Problems and Perspectives. Phys. Chem. Chem. Phys. 2012, 14, 13502.

(5) Vanin, M.; Mortensen, J. J.; Kelkkanen, A. K.; Garcia-Lastra, J. M.; Thygesen, K. S.; Jacobsen, K. W. Graphene on Metals: A van Der Waals Density Functional Study. Phys. Rev. B 2010, 81, 081408 .

(6) Seo, M. H.; Choi, S. M.; Kim, H. J.; Kim, W. B. The Graphene-Supported Pd and Pt Catalysts for Highly Active Oxygen Reduction Reaction in an Alkaline Condition. Electrochem. commun. 2011, 13, 182-185.

(7) Hsieh, S. H.; Hsu, M. C.; Liu, W. L.; Chen, W. J. Study of Pt Catalyst on Graphene and Its Application to Fuel Cell. Appl. Surf. Sci. 2013, 277, 223-230.

(8) Yoo, E.; Okata, T.; Akita, T.; Kohyama, M.; Nakamura, J.; Honma, I. Enhanced Electrocatalytic Activity of Pt Subnanoclusters on Graphene Nanosheet Surface. Nano Lett. 2009, 9, 2255-2259.

(9) Chan, K. T.; Neaton, J. B.; Cohen, M. L. First-Principles Study of Metal Adatom Adsorption on Graphene. Phys. Rev. B 2008, 77, 235430.

(10) Liu, X.; Wang, C. Z.; Yao, Y. X.; Lu, W. C.; Hupalo, M.; Tringides, M. C.; Ho, K. M. Bonding and Charge Transfer by Metal Adatom Adsorption on Graphene. Phys. Rev. B 2011, 83, 235411.

(11) Pinto, H.; Markevich, A. Electronic and Electrochemical Doping of Graphene by Surface Adsorbates. Beilstein J. Nanotechnol. 2014, 5, 1842-1848.

(12) Chen, J.-H.; Jang, C.; Adam, S.; Fuhrer, M. S.; Williams, E. D.; Ishigami, M. ChargedImpurity Scattering in Graphene. Nature Phys. 2008, 4, 377-381.

(13) Gierz, I.; Riedl, C.; Starke, U.; Ast, C. R.; Kern, K. Atomic Hole Doping of Graphene. Nano Lett. 2008, 8, 4603-4607.

(14) Pi, K.; McCreary, K. M.; Bao, W.; Han, W.; Chiang, Y. F.; Li, Y.; Tsai, S.-W.; Lau, C. N.; Kawakami, R. K. Electronic Doping and Scattering by Transition Metals on Graphene. Phys. Rev. B 2009, 80, 075406.

(15) Liu, X.; Wang, C. Z.; Hupalo, M.; Lu, W. C.; Tringides, M. C.; Yao, Y. X.; Ho, K. M. Metals on Graphene: Correlation between Adatom Adsorption Behavior and Growth Morphology. Phys. Chem. Chem. Phys. 2012, 14, 9157. 
(16) Krasheninnikov, A. V.; Lehtinen, P. O.; Foster, A. S.; Pyykkö, P.; Nieminen, R. M. Embedding Transition-Metal Atoms in Graphene: Structure, Bonding, and Magnetism. Phys. Rev. Lett. 2009, 102, 126807.

(17) Kaukonen, M.; Krasheninnikov, A. V.; Kauppinen, E.; Nieminen, R. M. Doped Graphene as a Material for Oxygen Reduction Reaction in Hydrogen Fuel Cells: A Computational Study. ACS Catal. 2013, 3, 159-165.

(18) Lu, Y.-H.; Zhou, M.; Zhang, C.; Feng, Y.-P. Metal-Embedded Graphene: A Possible Catalyst with High Activity. J. Phys. Chem. C 2009, 113, 20156-20160.

(19) Rodríguez-Manzo, J. A.; Cretu, O.; Banhart, F. Trapping of Metal Atoms in Vacancies of Carbon Nanotubes and Graphene. ACS Nano 2010, 4, 3422-3428.

(20) Gan, Y.; Sun, L.; Banhart, F. One- and Two-Dimensional Diffusion of Metal Atoms in Graphene. Small 2008, 4, 587-591.

(21) Wang, H.; Wang, Q.; Cheng, Y.; Li, K.; Yao, Y.; Zhang, Q.; Dong, C.; Wang, P.; Schwingenschlögl, U.; Yang, W.; et al. Doping Monolayer Graphene with Single Atom Substitutions. Nano Lett. 2012, 12, 141-144.

(22) Robertson, A. W.; Montanari, B.; He, K.; Kim, J.; Allen, C. S.; Wu, Y. A.; Olivier, J.; Neethling, J.; Harrison, N.; Kirkland, A. I.; et al. Dynamics of Single Fe Atoms in Graphene Vacancies. Nano Lett. 2013, 13, 1468-1475.

(23) Perdew; Burke; Ernzerhof. Generalized Gradient Approximation Made Simple. Phys. Rev. Lett. 1996, 77, 3865-3868.

(24) Troullier, N.; Martins, J. L. Efficient Pseudopotentials for Plane-Wave Calculations. II. Operators for Fast Iterative Diagonalization. Phys. Rev. B 1991, 43, 8861-8869.

(25) Soler, M.; Artacho, E.; Gale, J. D.; Garc, A.; Junquera, J.; Ordej, P.; Daniel, S. The SIESTA Method for Ab Initio Order- N Materials. J. Phys. Condens. Matter 2002, 14, 2745-2779.

(26) Monkhrost, H. J.; Pack, J. D. Special Points for Brillouin-Zone Integrations. Phys. Rev. B $1976,13,5188$.

(27) Grimme, S. Semiempirical GGA-Type Density Functional Constructed with a LongRange Dispersion Correction. J. Comput. Chem. 2006, 27, 1787-1799.

(28) Zobelli, A.; Gloter, A.; Ewels, C. P.; Seifert, G.; Colliex, C. Electron Knock-on Cross Section of Carbon and Boron Nitride Nanotubes. Phys. Rev. B 2007, 75, 245402.

(29) Skowron, S. T.; Lebedeva, I. V; Popov, A. M.; Bichoutskaia, E. Approaches to Modelling Irradiation-Induced Processes in Transmission Electron Microscopy. Nanoscale 2013, 5, 6677.

(30) Meyer, J. C.; Eder, F.; Kurasch, S.; Skakalova, V.; Kotakoski, J.; Park, H. J.; Roth, S.; Chuvilin, A.; Eyhusen, S.; Benner, G.; et al. Accurate Measurement of Electron Beam Induced Displacement Cross Sections for Single-Layer Graphene. Phys. Rev. Lett. 2012, 108, 196102.

(31) Santana, A.; Zobelli, A.; Kotakoski, J.; Chuvilin, A.; Bichoutskaia, E. Inclusion of 
Radiation Damage Dynamics in High-Resolution Transmission Electron Microscopy Image Simulations: The Example of Graphene. Phys. Rev. B 2013, 87, 094110.

(32) McKinley, W. A.; Feshbach, H. The Coulomb Scattering of Relativistic Electrons by Nuclei. Phys. Rev. 1948, 74, 1759-1763.

(33) Susi, T.; Kotakoski, J.; Arenal, R.; Kurasch, S.; Jiang, H.; Skakalova, V.; Stephan, O.; Krasheninnikov, A. V.; Kauppinen, E. I.; Kaiser, U.; et al. Atomistic Description of Electron Beam Damage in Nitrogen-Doped Graphene and Single-Walled Carbon Nanotubes. ACS Nano 2012, 6, 8837-8846.

(34) Susi, T.; Kotakoski, J.; Kepaptsoglou, D.; Mangler, C.; Lovejoy, T. C.; Krivanek, O. L.; Zan, R.; Bangert, U.; Ayala, P.; Meyer, J. C.; et al. Silicon-Carbon Bond Inversions Driven by 60-keV Electrons in Graphene. Phys. Rev. Lett. 2014, 113, 115501. 